\title{
Clinical application of a computerized system for physician order entry with clinical decision support to prevent adverse drug events in long-term care
}

\author{
Paula A. Rochon, Terry S. Field, David W. Bates, Monica Lee, Linda Gavendo, \\ Janet Erramuspe-Mainard, James Judge, Jerry H. Gurwitz
}

A simple prescribing decision can initiate unforeseen outcomes. The case described in Box I exemplifies how a cascade of unintended events can end in the development of a serious adverse drug event and in the elimination from a patient's medication regimen of a therapy that could have been beneficial if used safely.

Evidence obtained in the acute care hospital sector ${ }^{1}$ suggests that drug safety can be improved by means of computa erized physician order entry (CPOE) with clinical decision support (CDS). Such improvements might be particularly important in long-term care.

CPOE-CDS is an application that physicians use to enter patient prescriptions directly into a computer and receive immediate feedback about clinically important information that may improve their prescribing decision. ${ }^{2,3}$ Specifically, an au-

Box 1: Case report

An 89-year-old woman living in a long-term care facility had a medical history of atrial fibrillation, stroke, dementia and hypertension. Her medications included warfarin, the dosage of which her primary care provider has been adjusting to maintain her international normalized ratio (INR) in the range of 2.0-3.0.

When a fever developed, a covering physician prescribed cephalexin ( $500 \mathrm{mg}$ by mouth, 4 times daily for $7 \mathrm{~d}$ ) for a presumed urinary-tract infection. The next morning (day 2), her primary care physician was telephoned with the results of a day 1 test: INR 1.75. He accordingly increased the patient's daily dose of warfarin from $4 \mathrm{mg}$ to $5 \mathrm{mg}$. He was not told about the cephalexin ordered the previous evening by the covering physician.

One week later, the patient's INR was 13.8; another covering physician was notified. That day, warfarin was withheld. The following day, her INR rose to 16.1 ; she continued to receive no warfarin, and no vitamin $\mathrm{K}$ was administered.

The next day, the patient became short of breath. When chest radiography revealed an infiltrate, the covering physician ordered amoxicillin/clavulanate $(875 \mathrm{mg}$ by mouth every 12 hours for 10 days). The next day, she passed tarry stool; omeprazole was initiated empirically.

The following morning, the patient's hemoglobin measurement was $83 \mathrm{~g} / \mathrm{L}$ (hematocrit 0.25) and her INR, 11.3. The primary care physician was notified, and the patient received $10 \mathrm{mg}$ of vitamin $\mathrm{K}$ by injection, which resulted in a decline in her INR to 0.9 . The physician wrote that warfarin would not be reinitiated in this patient because "anticoagulation has been difficult to control, for unclear reasons." tomated interface links the newly ordered drug therapy to clinical, drug and laboratory information in a way that guides prescription decisions in the direction of increased safety and ultimately reduces the medication errors that lead to adverse events. ${ }^{4}$ For example, if a physician enters an order for an antidepressant drug for a patient already taking multiple psychoactive medications, the CDS system would display a warning about the potential for oversedation, confusion, delirium and falls. It would then advise the physician to evaluate the need for each psychoactive medication, indicate those already prescribed and suggest prescribing the lowest feasible dose of the new drug.

CPOE-CDS is being used with success in acute care facilities. Use of CPOE was recommended in a 1999 Institute of Medicine report on reducing medical error, and one study ${ }^{4}$ found that CPOE reduced serious medication errors by $55 \%$. In 2000, the State of California passed a bill (California Senate Bill I875) mandating that urban hospitals implement technological programs to reduce error (e.g., CPOE) by 2005 . Implementation of such applications has been recommended as well by such US organizations as the Leapfrog Group (www.leapfroggroup.org) and the National Quality Forum (www.qualityforum.org).

New strategies are needed to improve prescribing practices in extended care. On average, residents of long-term care facilities are prescribed more than 6 concurrent drug therapies. Such a high frequency of drug combinations among people of advanced age and who are often in frail condition markedly increases their potential for adverse events. When Gurwitz and colleagues ${ }^{5}$ evaluated adverse drug events in 18 such facilities in the United States, monitoring nearly 3000 people for a total of 28839 resident-months, they documented 546 adverse events. ${ }^{5}$ Accordingly, in a roo-bed long-term care facility, about 2 adverse drug events could reasonably be expected to occur per month; but because the investigators considered only the adverse events documented in medical charts, ${ }^{5}$ their estimate was probably conservative. More recently, when Gurwitz's group ${ }^{6}$ did detailed chart reviews and used computer-generated signals to help identify adverse drug events, the rates they found were 5 times as high. This finding suggests that previous studies have likely underestimated the true rate of adverse events in the setting of longterm care.

Most prescribing errors are made at the ordering and monitoring phases of the process of pharmacotherapeutic care. ${ }^{5,7} \mathrm{~A}$ flowchart illustrating these errors in the case report can be found as an online figure (available at www.cmaj.ca 
/cgi/content/full/I74/I/52/DCI). Errors I and 2 occurred at the ordering stage. The covering physician prescribed cephalexin without considering that the patient was taking warfarin, a drug that interacts with cephalexin to increase international normalized ratios (INRs); the potential interaction was not noticed when the new therapy was ordered (error I). When the primary care provider increased the dose of warfarin, he was unaware that the covering physician had prescribed cephalexin (error 2).

Errors 3 and 4 occurred at the monitoring stage. The physician failed to order vitamin $\mathrm{K}$ in response to an elevated INR (error 3). Close monitoring was not provided after amoxicillin/clavulanate and warfarin were ordered, which are known to interact (error 4), and which led to an elevated INR.

Error 5 occurred at the ordering stage again, when the physician ordered omeprazole in response to a gastrointestinal bleed without considering that the bleed may have been related to the elevated INR. Error 6 was to consider that the patient had been hard to manage, when in fact the issues were predictable.

Let us now consider what might have happened to the patient had the physicians entered the drug orders directly into a computer using CPOE-CDS (Box 2). A key advantage of CPOE is that decision support (Fig. I) can be provided when drug therapies are ordered, even remotely. As the revised scenario in Box 2 indicates, had the physicians in question had access to a CPOE-CDS system, the patient's outcome might have been quite different.

Box 2: How the use of a CPOE-CDS system might have changed the outcome of the case report

An on-call physician is contacted by phone by the registered nurse on duty and asked to order a prescription for the patient for a urinary-tract infection. From home, the on-call physician goes to his computer, logs in to the secure CPOE system and enters an order to prescribe cephalexin.

A CDS warning, as shown in Fig. 1, appears on the computer screen stating that this patient is taking warfarin, which may interact with cephalexin to produce an elevated INR. The system suggests that the physician order a test for INR level in 3 days and to decrease the recommended dose for the warfarin. Accordingly, the physician orders a follow-up INR test and lowers the maintenance dose.

The next morning, the primary care physician is called with the results of the initial blood tests, which report an INR of 1.75. Looking at the patient's medication list through the CPOE system, he sees that the cephalexin has been added, the warfarin dose has been decreased, and a repeat INR test has been ordered. The INR returns at 2.2. No additional warfarin is prescribed, and the INR continues to be monitored.

A week later, her INR is reported to be 3.2 . The primary care physician decreases the dose of warfarin further for several days, then increases it back to the dose the patient had been taking before the cephalexin was given. Her INR readings stay between 2 and 3 .

Note: $\mathrm{CPOE}=$ computerized physician order entry, $\mathrm{CDS}$ = clinical decision support, INR = international normalized ratio.

\section{Box 3: Key points}

- Adverse drug events are common in long-term care

- Most errors occur at the ordering and monitoring stages of the prescribing process

- Computerized physician order entry with clinical decision support has the potential to reduce medication errors, which could lead to a decrease in adverse events

The age and health of residents of long-term care facilities and their use of multiple drug therapies ${ }^{8}$ increases the likelihood of adverse events. The staffing structure at such facilities - specifically, limited access to physician time and relatively few registered nurses on staff - may also contribute to the problem. Because many of the physicians on call work there only part-time, prescribing decisions are often made on the telephone without easy access to relevant facts such as concurrent medication use or laboratory data. Information important to prescribing decisions may therefore not be known when a new drug is prescribed. Given all these factors, CPOE implementation in these settings is a logical next step. The fact that the number of long-term care beds exceeds those in acute care facilities underscores the importance of implementing CPOE systems to reduce adverse drug events in this population.

We believe that CPOE in the long-term care setting is a realistic and desirable option (Box 3). Smaller long-term care facilities are often part of larger organizations that work with a defined group of pharmacies and laboratories, which provides the opportunity to create systems across the organization. Given the high rates of adverse drug events reported among residents of long-term care facilities ( 9.8 per Ioo resident-months) and that more than $40 \%$ of these are judged to be preventable, ${ }^{6}$ system-level approaches are required to guide appropriate prescribing decisions. We are currently aware of 2 long-term care facilities, Baycrest in Toronto and the Masonicare Facility in Connecticut, with CPOE-CDS systems in place. These institutions may serve as models for other long-term care settings as they develop their systems. CPOE systems will need to start by building on the limited technological infrastructure that exists in many long-term care facilities. The initial steps in this process will be to find ways to link the provider, pharmacy and laboratory systems. This will lay groundwork for the widespread implementation of CPOE.

A CPOE system can also cause errors. ${ }^{3}$ Koppel and coworkers ${ }^{9}$ conducted a study of providers working with a $\mathrm{CPOE}$ system at an acute care teaching hospital. Using qualitative and quantitative approaches, they identified 22 types of medication error risks. These included fragmented display of orders, pharmacy inventory display of drug doses that were confused with recommended dosages, and ignored renewal orders for antibiotic therapy when notices were placed in the paper chart and not in the computer. These findings indicate the need to continually improve the CPOE system when medication error risks are identified. 


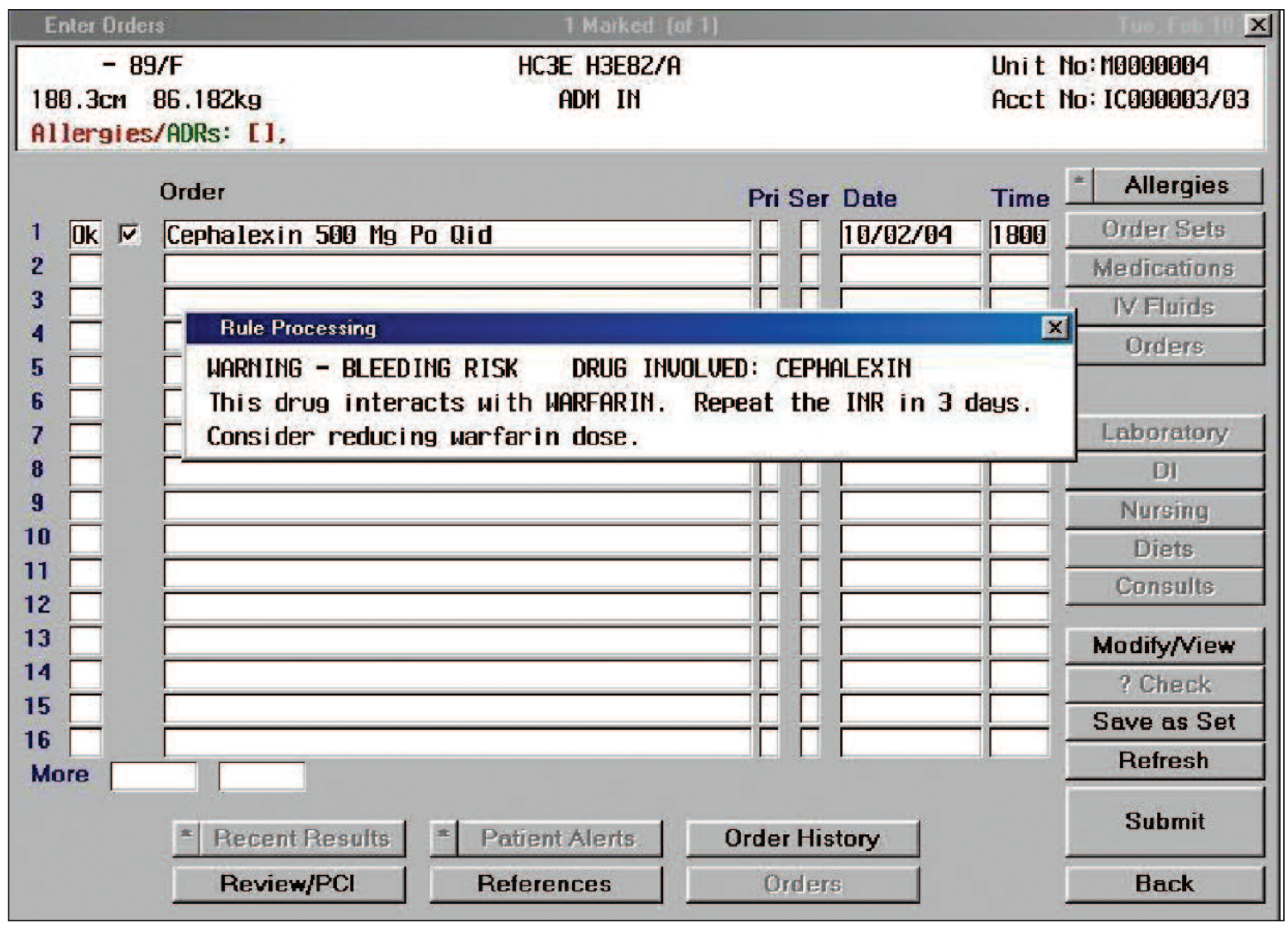

Fig. 1: Computer screen showing a warning about drug interaction between warfarin and cephalexin.

The case reported illustrates how a simple prescribing decision in the long-term care setting can lead to a cascade of errors culminating in an adverse drug event. Had a computerized system been in place, such prescribing errors could have been avoided. CPOE-CDS is a promising new technology that may be very useful in the setting of longterm care.

This article has been peer reviewed.

From the Kunin-Lunenfeld Applied Research Unit (Rochon, Lee), Baycrest (Gavendo, Erramuspe-Mainard), Toronto, Ont.; the Meyers Primary Care Institute, Fallon Foundation and University of Massachusetts Medical School (Field, Gurwitz), Worcester, Mass.; the Division of General Internal Medicine, Brigham and Women's Hospital, and Harvard Medical School (Bates), Boston, Mass.; and Evercare Connecticut (Judge), Hartford, Conn.

Competing interests: None declared for Paula Rochon, Terry Field, Monica Lee, Linda Gavendo, Janet Erramuspe-Mainard, James Judge and Jerry Gurwitz. David Bates has received speaker's fees from the Eclipsys Corporation, which has licensed the rights to the Brigham and Women's Hospital Clinical Information System (the hospital no longer has a financial relationship with Eclipsys). He is a coinventor on Patent No. 6029138 held by the hospital on the use of decision-support software for medical management, licensed to the Medicalis Corporation. He also holds a minority equity position in the privately held company Medicalis, which develops Web-based decision support for radiology test ordering, and he serves as a consultant to Medicalis.

Contributors: Paula Rochon designed and conceived the project and wrote the first draft of the manuscript. Terry Field, David Bates, Monica Lee, James Judge and Jerry Gurwitz assisted in the design of the project and revised the manuscript. Linda Gavendo and Janet Erramuspe-Mainard made critical revisions to the manuscript.
Acknowledgements: We thank the Computerized Physician Order Entry Team at Baycrest for their work: Joel Abrams, Aidlee Craft, Sid Feldman, Michael Gilman, Michael Gordon, Sheldon Hershkop, Ella Iousoupova, Sheryl Korn, Joanna Mills, Hasmukh Parmar, Vic Lambert, Jacqui Phillips, Joy Richards, Sam Rubenzahl, Cristina Scherf, Linda Trick, Jane Uyger and Sabiya Yan. We also thank Jennifer Gold for her assistance with the preparation of the manuscript.

This project was funded by research grants from the Agency for Healthcare Research and Quality (HSoro48I and I ROI HSI5430-oI). The contents are solely the responsibility of the authors and do not necessarily represent the official views of the Agency for Healthcare Research and Quality.

\section{REFERENCES}

I. Briggs B. CPOE: order from chaos. Health Data Manag 2003;11:44-8.

2. Doolan DF, Bates DW, James BC. The use of computers for clinical care: a case series of advanced US sites. J Am Med Inform Assoc 2003;10:94-107.

3. Wears RL, Berg M. Computer technology and clinical work: still waiting for Godot. JAMA 2005;293:I26I-3.

4. Bates DW, Leape LL, Cullen DJ, et al. Effect of computerized physician order entry and a team intervention on prevention of serious medication errors. JAMA I998;280:13II-6.

5. Gurwitz JH, Field TS, Avorn J, et al. Incidence and preventability of adverse drug events in nursing homes. Am J Med 2000;109:87-94.

6. Gurwitz JH, Field TS, Judge J, et al. The incidence of adverse drug events in two large academic long-term care facilities. Am J Med 2005;II8:25I-8.

7. Gurwitz JH, Field TS, Harrold LR, et al. Incidence and preventability of adverse drug events among older persons in the ambulatory setting. JAMA 2003;289:II07-I6.

8. Field TS, Gurwitz JH, Avorn J, et al. Risk factors for adverse drug events among nursing home residents. Arch Intern Med 200I;161:1629-34.

9. Koppel R, Metlay JP, Cohen A, et al. Role of computerized physician order entry systems in facilitating medication errors. JAMA 2005;293:II97-203.

Correspondence to: Dr. Paula Ann Rochon, Baycrest KuninLunenfeld Applied Research Unit, 3560 Bathurst St., Toronto ON M6A 2EI; fax 4I6 785-2378; paula.rochon@utoronto.ca 